\title{
Bridging mechanisms of through-thickness reinforcement in dynamic mode I\&II delamination
}

\author{
Hao Cui ${ }^{\mathrm{a} 1}$, Mehdi Yasaee ${ }^{\mathrm{b}}$, Gordon Kalwak ${ }^{\mathrm{a}, \mathrm{c}}$, Antonio Pellegrino ${ }^{\mathrm{a}}$, Ivana K. \\ Partridge $^{b}$, Stephen R. Hallett ${ }^{b}$, Giuliano Allegri ${ }^{d}$, Nik Petrinic ${ }^{a}$ \\ ${ }^{a}$ Department of Engineering Science, University of Oxford, Oxford, UK \\ ${ }^{\mathrm{b}}$ Advanced Composites Centre for Innovation and Science (ACCIS), \\ University of Bristol, Bristol, UK \\ ${ }^{\mathrm{c} R o l l s-R o y c e ~ p l c, ~ D e r b y, ~ U K ~}$ \\ ${ }^{\mathrm{d}}$ Faculty of Aeronautics, Imperial College of London, London, UK
}

\begin{abstract}
Z-pin through-thickness reinforcement is used to improve the impact resistance of composite structures; however, the effect of loading rate on Z-pin behaviour is not well understood. The dynamic response of Z-pins in mode I and II delamination of quasiisotropic IM7/8552 laminates was characterized experimentally in this work. Z-pinned samples were loaded at both quasi-static and dynamic rates, up to a separation velocity of $12 \mathrm{~m} / \mathrm{s}$. The efficiency of Z-pins in mode I delamination decreased with loading rate, which was mainly due to the change in the pin misalignment, the failure surface morphology and to inertia. The Z-pins failed at small displacements in the mode II loading experiments, resulting in much lower energy dissipation in comparison with the mode I case. The total energy dissipation decreased with increasing loading rate, while enhanced interfacial friction due to failed pins may be largely responsible for the higher energy dissipation in quasi-static experiments.
\end{abstract}

\footnotetext{
${ }^{1}$ Corresponding author: email: hao.cui@eng.ox.ac.uk;

Phone: $+44(0) 1865613452$
} 


\section{Keywords}

Z-pin; mode I; mode II; delamination; dynamic

\section{Introduction}

Carbon fibre reinforced polymer matrix composites are extensively used in the aerospace industry, due to their high in-plane strength, stiffness and low weight. However, the absence of reinforcement in the through-thickness direction has led to delamination becoming one of the dominating failure modes for composite laminates[1, 2]. Z-pinning has been developed as a cost-effective method to improve the resistance to delamination growth, using metallic or carbon fibre rods inserted into the lay-up before curing [3, 4].

The Z-pins are very effective in increasing the delamination toughness in quasi-static tests [5-8], and they have shown superior performance in mode I delamination compared with mode II case [9-11]. In mode I delamination, the Z-pins are normally pulled out, and considerable energy is dissipated during the frictional pull-out process $[5,12]$. In contrast, the Z-pins tend to split and rupture when loaded in shear, offering only relatively low energy dissipation $[6,9,10,13]$. The bridging responses of Z-pins, i.e. the evolution of bridging force with the relative displacement of the delamination surface, is essential for analysing the failure of Z-pinned composites [14, 15].

The failure mechanisms of a Z-pin also heavily depend on the topology of its surroundings. The layup of composite laminates [10], the insertion angle of Z-pins [9] and also the insertion length [12] have significant influence on the failure modes and energy dissipation of Z-pins. The Z-pins are surrounded by resin pocket in laminates [3], and the resin may get deformed and damaged due to lateral deformation of the pins [16]. The resin material [17], and the composite laminate [18] are both very sensitive to strain 
rate, and their strength increases considerably with loading rate. Accordingly, the mechanical performance of the pins can be expected to show some dependency on the loading rate.

Composite structures may experience various impact threats during service, and extensive delamination may be introduced as a result $[19,20]$. Z-pins are therefore used to prevent extensive delamination and thus overall catastrophic failure [21, 22] in structures exposed to impact threats. Despite comprehensive studies on the Z-pin behaviour in quasi-static rate, very few works addressing the dynamic performance of Zpins exist in open literature. The mode I delamination of Z-pinned laminated was tested in [23] at two different velocities: $1 \mathrm{~mm} / \mathrm{min}$ and $100 \mathrm{~mm} / \mathrm{min}$. The efficiency of Zpinning increased with loading rate for large pins because of enhanced interfacial friction, whilst it decreased for small diameter pins as pins ruptured at increased loading rate; this study indicated a noticeable dependency of the Z-pin response on loading rate. A much higher loading rate was achieved by loading Z-pinned Double Cantilever Beam (DCB) samples with a flying wedge $[24,25]$; the delamination toughness was found to decrease with increased loading rate ranging from quasi-static to $40 \mathrm{~m} / \mathrm{s}$, while it started to increase again at a still higher rate $(50 \mathrm{~m} / \mathrm{s})$. The contribution of Z-pins to delamination resistance is challenging to quantify in these tests as kinetic energy may dominate the fracture process of the DCB samples if the crack velocity is high[26].

The dynamic bridging response of Z-pins is essential for the design, analysis and certification of composite structures threatened with impact loading[27]. The aim of the work presented in this paper has been to experimentally characterize the mechanical response of Z-pins, and understand their failure mechanisms at different loading rate. This will contribute to the ability to better design impact resistant structures with Z-pin 
reinforcement and also create a comprehensive experimental database for the development and validation of predictive numerical analysis tools. Z-pins embedded in pre-delaminated laminates were tested at a variety of loading rates from $0.01 \mathrm{~mm} / \mathrm{s}$ to $12 \mathrm{~m} / \mathrm{s}$, and for the first time the dynamic performance of Z-pins was characterized using the split Hopkinson bar apparatus, allowing accurate traction-displacement curve extraction.

\section{Experiments}

\subsection{Specimen configuration}

Z-pins of $0.28 \mathrm{~mm}$ diameter made from T300/BMI material were used to reinforce quasi-isotropic laminates. The laminates were made from Hexcel's IM7/8552 pre-preg material. A layer of PTFE film was inserted at the mid-plane of the layups, to create a pre-existing delamination and ensure that the measured bridging force was purely from the Z-pins. The layup in the top half was $[0 / 45 / 90 /-45]_{4 S}$, and the bottom half was $[90 /-45 / 0 / 45]_{4 s}$. This quasi-isotropic layup was chosen to represent the multi-directional laminates commonly used in industrial applications and has a $90^{\circ}$ interface at the midplane to avoid nesting of the plies. Non unidirectional stacking sequences have been shown to have strong influence on altering the Z-pin bridging response, relative to unidirectional laminates [10].

The pull-out force exerted by a single pin is relatively low [10], and quite challenging to measure in a dynamic test. To overcome this, an array of $4 \times 4$ pins was inserted in each specimen in this investigation. An averaged force was then calculated to represent the properties of a single pin. Specimens were machined from the pinned laminates, to make 
10x10mm blocks, with thickness of $8 \mathrm{~mm}$. A $2 \%$ Z-pin areal density was used in the pinning process, and the distribution of Z-pins over the specimen is shown in Fig.1.

The Z-pins were not perfectly vertical to the laminate plane due to the limits of manufacturing capability. As illustrated in Fig.1, all pins were misaligned predominantly in the same direction giving a uniform misalignment angle. The average misalignment angle of $4 \times 4$ pins in each specimen was measured as around $9 \pm 3^{\circ}$ for all samples. The shear failure of Z-pins may be sensitive to the misalignment angle, and the Z-pins were loaded with the nap[10] in all shear tests.

\subsection{Test setup}

Aluminium fixtures were designed to apply tension and shear displacement load to the z-pinned samples, as presented in Fig.2. The M6 thread on one end of these fixtures was screwed directly on to the testing machines. 3M Scotch-Weld DP490 adhesive was used to bond the samples to the aluminium blocks. The mode II specimens were constrained within a brass sleeve to avoid lateral opening displacements, which in turn ensured pure mode II delamination behaviour. Quasi-static tests were performed using a Zwick testing machine with loading rate of $0.01 \mathrm{~mm} / \mathrm{s}$. A split Hopkinson tension bar system was used for the dynamic tests, with the striker velocity reaching up to $12 \mathrm{~m} / \mathrm{s}$ in this investigation. The failure process was monitored with high speed cameras as illustrated in Fig.3. The specimen surface was painted with black speckles on a white back ground, enabling the digital image correlation (DIC) method to be used to track the opening and shear displacement of the specimen.

The failure process tends to be unstable in quasi-static shear tests, and the sharp drop in load of the Z-pin response was not able to be captured at the acquisition rate of the 
built in load cell connected to the screw-driven machine. To overcome this limitation, in the quasi-static experiment, an instrumented low mechanical impedance aluminium tube, with a strain gauge mounted on its surface for measurement, was connected between the loading cell and the sample. The tube was $0.9 \mathrm{~m}$ long, to ensure that the entire drop of the bridging force could be measured before the reflected wave from the load cell end reached the strain gauge. The strain gauge on this tube was connected to the signal conditioners and oscilloscopes and was used to measure the strain wave during the unstable failure process at an acquisition rate of $5 \mathrm{MHz}$, allowing the measurement of the bridging force. A high-speed camera was triggered at the initiation of damage, to record the displacement during the unstable failure event.

The split Hopkinson tensile bar system used in dynamic tests is illustrated in Fig.3b. Detailed information on the apparatus can be found in [28]. The long projectile can generate stress pulse with duration of $1 \mathrm{~ms}$, allowing the Z-pin to be completely pulled out in a single pulse at modest velocity. The strain gauge attached on bars were used to measure the strain during the test, and then used to calculate the force and displacement of the samples.

\section{Numerical model for inertia effect}

The force measured with strain gauges on the Hopkinson bars is valid for calculating the force on the sample only if there is no inertia effect from the samples $[29,30]$. Dynamic equilibrium conditions are difficult to achieve during dynamic shear tests, as failure occurs at very small displacements. Besides, a relatively heavy fixture is used; introducing a considerable inertia effect into the measured force. In the quasi-static mode II tests, the Z-pin failed very rapidly once damage initiated, and the sharp drop of bridging 
force may result in acceleration of the aluminium fixture. Consequently, the effect of inertia should also be considered.

A linear elastic model was built in Abaqus 6.14, to evaluate the influence of inertia, and to explore a method for calibrating the experimental results. The aluminium tube output bar used in experiments was modelled as shown in Fig.4, with the attached aluminium fixture and half of the laminated sample. The bridging force of Z-pins was modelled with surface traction stress on the laminates in this simulation. As shown in Fig.4, the response of Z-pins in dynamic simulation was assumed to be bilinear, with the linear descending behaviour of the Z-pins after damage initiation in quasi-static tests.

The strain on an element $200 \mathrm{~mm}$ from the end of the bar, representing the measured strain from strain gauge in experiments, was used to calculate the force carried by the specimen, representing the force measurement in experiments:

$$
F_{m}=\varepsilon E \psi
$$

where $E$ is the Young's modulus of Aluminium, $\varepsilon$ is the longitudinal elastic strain of the bar, and $\psi$ is the cross-sectional area of the tube.

The Abaqus/Explicit analysis was used in the simulation of dynamic tests, and the force calculated with $\mathrm{Eq}(1)$ was then compared against the input shown in Fig.4. For the simulation of quasi-static configuration, the Abaqus/Standard analysis step was conducted first to achieve equilibrium of stress distribution within the whole system. In the following Abaqus/Explicit step, the bridging force descended to zero within $0.1 \mathrm{~ms}$, and the strain history on the aluminium tube was used to calculate the force with $\mathrm{Eq}(1)$.

The acceleration of the fixture during the fracture event was output, and the inertia force for accelerating the aluminium fixture and the specimen was calculated as: 


$$
F_{k}=A m
$$

where $m$ is the mass of the aluminium block and half of the specimen (6.5 g in total), assuming them as concentrated mass. The acceleration $A$ of the fixture and half specimen is assumed to be uniform across it and is obtained directly as output from the FEM package. The actual force acting on the Z-pinned interface was then corrected with:

$$
F=F_{m}+F_{k}
$$

where $F_{m}$ is the force evaluated from the strain signal on the bar.

\section{Results and discussions}

\subsection{Inertia effect}

The force measured in the finite element analysis from the strain on the bar and the actual force applied to the sample in the numerical simulations is plotted in Fig.5. The force measurement using strain on the bar underestimated the force of the raising edge, while overestimated the falling edge due to the inertia effect shifting the curve to the right. This force was corrected with the inertia force from the fixture, and good correlation was achieved with the input data. This numerical study confirmed that the inertia effect may influence the measurement of force, and proper correction should be considered in dynamic tests. The area under the load-displacement curves, namely the energy dissipation of the Z-pins in experiments, was not noticeably affected by the inertia effect in dynamic tests, while was significantly raised in quasi-static tests. The amount of extra energy in original measurements was mainly caused by the kinetic energy of the fixture, as confirmed in Fig.5b. 


\subsection{Mode I behaviour}

\subsubsection{Bridging response}

The failure process was recorded with high-speed imaging for all tests. All Z-pins were pulled out from laminates regardless of the loading rate, as shown in Fig.6. The bridging force, the velocity and the displacement of Z-pinned samples in dynamic mode I tests were calculated via unidimensional stress wave analysis[28]. As shown in Fig.7a, the bridging force raised to its peak in the first $0.1 \mathrm{~ms}$ when the displacement was still increasing in nonlinear manner, indicating the inertia effect at this stage should be considered. The energy dissipation within this stage was small due to the small displacement, which was not influenced noticeably by the inertia effect either, as proven in Fig.5a. The Z-pins were pulled out at an almost constant rate after $0.1 \mathrm{~ms}$, until the complete failure.

The mode I bridging forces at three different loading rates are plotted in Fig. $7 \mathrm{~b}$ as a function of opening displacement. The bridging forces first increased with displacement until their maxima, and then followed with sharp drops. The force drop brings in inertia effect in both dynamic and quasi-static experiments. However, it is very challenging to estimate this inertia effect in dynamic tests, as it requires very high frame rate to capture the inertia effect due to the force drop, while then the whole failure process would require more frames than that our current camera could provide. Since the energy dissipation during this pin- matrix failure stage is much smaller than that in the frictional pulling out stage, the inertia effect in mode I tests is neglected in this work. The peak bridging force in dynamic tests was slightly higher than that in quasi-static tests, although considerable scatter was noticed. Considering the fact that the peak forces in dynamic tests were 
underestimated due to inertia effect, the maximum bridging forces may increase with loading rate more significantly than shown in Fig. $7 \mathrm{~b}$. The sharp drop after peak force is indicative of the fracture of the pin-composite bond, and the significant rate dependence of matrix dominated shear strength [31] is likely to be responsible for the higher of peak bridging force in dynamic tests. All Z-pins were pulled out gradually after this interfacial failure. During this pull-out, the Z-pins experienced a nonlinear increase in the bridging force in quasi-static tests, while the bridging force decreased almost linearly with displacement in dynamic tests. The mode I samples featured higher bridging force for initiation compared with previous work [10], indicating the bonding between Z-pin and laminates has improved [32].

\subsubsection{Failure mechanisms}

All Z-pins were pulled out during the mode I tests, while their bridging response showed significant rate dependency. The Z-pins were investigated with scanning electron microscopy (SEM) after being pulled out, and their failure surfaces were compared with original Z-pins in Fig.8. A smooth surface similar to that of original pins was observed for all pins pulled out at different rates, indicating the debonding of Z-pins with surrounding laminates. There was however some additional inter-fibre failure and fibre rupture within the tested Z-pins from all experiments, which were not present in the untested pins. There were no obvious characteristics of the surface condition of the Zpins that could be said to have been significantly influenced by loading rate.

After crack formation at the pin-laminate interface or within pins, the Z-pins were pulled out gradually from laminates. The bridging force during this stage is mainly attributed to the frictional shear stress at the interface between Z-pin and laminates [33]. 
It experienced a significant nonlinear increase in quasi-static tests as shown in Fig.7. The increase of bridging force was less significant in dynamic tests, and followed by linear decrease with displacement. As sketched in Fig.9a, the Z-pins were not perfectly perpendicular to the laminates in the specimens used here. During the pull-out process, the Z-pins may get bent, which results in extra pressure on the crack surface. Accordingly, enhanced frictional stress can form $[10,11,16]$, which in turn increase the bridging force during the pulling out process.

The holes vacated by Z-pins were studied to reveal the surface condition after the pull-out process, as shown in Fig.9a. The middle section of the hole (in blue rectangle) is most representative for the frictional pull-out process, as the interaction between the Zpin and the surrounding matrix can be clearly identified. In our study, two distinct zones around the circumference of the hole were defined: the opening side where the Z-pin may moves away from the surface, and the plough side where the Z-pins applied extra pressure.

The original fracture surface on the opening side was better maintained than that in the plough side, because of less frictional sliding with the pin. As shown in Fig.9b, the open side features distributed cusps as consequence of the shear dominated fracture. On the plough side, the cusps have been rubbed off, with only a smooth imprint left by the sliding of carbon fibres, suggesting heavy friction during pull-out process in this enhanced friction zone. The fracture surface in dynamic tests was less rough than that in quasi-static ones, because of the reduced ductility of epoxy with increase in strain rate. Micro cracks on the failure plane tend to be more closely spaced during dynamic fracture, and the size of shear cusps decreases due to the limited volume between neighbouring cracks[31]. This relatively smooth fracture surface may result in less frictional resistance at the pin-laminate interface. The surface morphology on both the opening and plough 
sides of the dynamic test were very similar to each other, suggesting not significant influence from enhanced friction zone. There is limit on the maximum frictional stress at the interface[34], and this maximum frictional stress may be dependent on the roughness of crack surface and the strain rate sensitivity of matrix material. The smooth surface in dynamic test may bring down this maximum frictional stress, and making the influence of this enhanced friction zone less significant in dynamic cases. The enhanced friction zone was considered to be responsible for the nonlinear bridging force in the quasi-static tests, and its absence in the dynamic tests may be the reason for the linear decrease of bridging force with pull-out displacement.

The mode I bridging response was influenced by several factors as presented in this section. As the fracture surface showed dependence on loading rate in Fig.9, the frictional stress at interface may vary. The lateral compression of the Z-pins on surrounding laminates, especially on the plough side as shown in Fig.9, will be dependent on the pin misalignment and loading rate. It is these factors that were thought to be responsible for the negative rate dependence of Z-pin efficiency in resisting mode I delamination.

\subsection{Mode II behaviour}

The failure process of Z-pinned laminates loaded in mode II was monitored with high speed video cameras, and presented in Fig.10. A brass sleeve was used during these tests to prevent induced bending and ensure pure shear loading on the Z-pins. The measured opening displacement of the Z-pinned interface was less than $0.01 \mathrm{~mm}$ before complete failure of the Z-pins, much less than the shear component. The relative displacement of both sides of the Z-pinned laminate was obtained by means of DIC analysis of the recorded images. It worth noting that there is a lateral force between the brass sleeve and cylindrical fixture, and additional frictional force may thus be involved in the measured 
Z-pin response. Since the frictional coefficient between the brass sleeve and aluminium fixture is low, this friction effect was not considered in this study.

The bridging force in quasi-static tests was recorded using two separate data acquisition systems as illustrated in Fig.3. A typical quasi-static mode II test result is shown in Fig.11a, the rising edge of the Z-pin response was captured by the Zwick testing machine load cell, whilst the falling edge was captured with the strain gauge on the bar and oscilloscope at a high sampling rate. The Z-pins experienced an almost linear rise of bridging force with shear displacement until about $0.15 \mathrm{~mm}$; the bridging force started to decrease nonlinearly after damage. The bridging force decreased to zero at a displacement of around $0.6 \mathrm{~mm}$. The inertia effect in quasi-static tests should also be considered, as the sample and attached fixture was accelerated when the bridging force dropped to zero within less than $1 \mathrm{~ms}$. The acceleration history during the fracture event could not be accurately determined due to the limited number of images recorded during the failure process, and the true bridging force after damage initiation was not possible to be produced. However, the number of recorded images was sufficient for an accurate compensation of the kinetic energy. The energy dissipation was calculated by integrating of area under the bridging force-displacement curve, and the kinetic energy of the aluminium fixture was corrected accordingly. The energy dissipation of Z-pins will be presented in Section 4.4.

The images taken during dynamic mode II tests were used to analyse the displacement of the aluminium fixtures attached to the input and output bars. Knowing the time interval between each image, the velocity can then be calculated and plotted as in Fig.11b. Both fixtures were accelerated as the stress wave propagated from the input bar to the output bar. The relative velocity between these two fixtures reached an almost constant value at 
around $0.06 \mathrm{~ms}$, starting to increase again at around $0.12 \mathrm{~ms}$ when the Z-pins started to fail. Although a relatively constant loading rate of about $1.5 \mathrm{~m} / \mathrm{s}$ was reached in these tests, the velocity of these fixtures kept increasing, indicating that the inertia force has influenced the original force measured by the Hopkinson bars. The acceleration of the sample and fixture can be estimated from the velocity-time curves illustrated in Fig.11b. The force of inertia, determined from the acceleration history, was then used to correct the force measured by the bars. As shown in Fig.11c, the bridging force showed a steeper rising edge after correction, and it reduced earlier than it appeared to in the original results.

The bridging response of the Z-pins at different loading rate is presented in Fig.11d. The Z-pins in dynamic tests showed higher initial stiffness than quasi-static ones, while the maximum bridging force was comparable at both loading rates. Accordingly, the efficiency of Z-pins in improving the delamination initiation is not noticeably influenced by loading rate. The Z-pins lost their loading capacity at about $0.2 \mathrm{~mm}$ displacement in dynamic tests, while the quasi-static behaviour features a relatively long falling edge of the bridging response, which could possibly be attributed to frictional forces at the interface.

The Z-pin fracture surfaces from both the quasi-static and dynamic shear tests are shown in Fig.12. All pins were ruptured near to the specimen mid-plane, with sparsely distributed splitting cracks between fibres. The debonding of Z-pins from laminates was observed, as well as the fibre-matrix failure within Z-pins. The fracture surface in dynamic tests was much flatter and more uniform than that in quasi-static tests. The rough fracture surface of Z-pins in the quasi-static tests, giving rise to frictional stress in addition to the bridging force, may be responsible for the higher load capacity after failure initiation than that in dynamic case. 


\subsection{Energy dissipation}

The energy dissipated during the pulling out process, which is the key property for enhancing the delamination resistance of composite laminates, was calculated from integration of the area under the bridging force-displacement curves. It is interesting to notice in Fig. 13 that the Mode I energy dissipation decreased with the increase in loading rate. The nominal improvement to the mode I delamination toughness of an unpinned composite laminate can be calculated by the energy dissipation of the Z-pins per unit laminate area. For the volume fraction of $2 \%$ in the specimens tested here, there is one pin per $1.75 \times 1.75 \mathrm{~mm}^{2}$. The apparent delamination toughness improvement with Zpinning is $34.1 \mathrm{~kJ} / \mathrm{m}^{2}$ at quasi-static rates, decreasing to $13.6 \mathrm{~kJ} / \mathrm{m}^{2}$ at a loading rate of $12 \mathrm{~m} / \mathrm{s}$. For the IM7/8552 material tested here, this represents a considerable increase from the low mode I delamination resistance of $\sim 0.2 \mathrm{~kJ} / \mathrm{m}^{2}$ [35]. The Z-pins in dynamic mode I delamination are thus still very effective, even though there is a reduction from the quasi-static rate tests. The mode II results are presented both with and without the energy absorbed after the peak load of the traction-displacement curve. The complete energy dissipation in quasi-static experiments was considerably higher than that in the dynamic case. The post-peak energy in quasi-static tests may be largely due to friction between the interfaces, which was enhanced by the failed pins. If one excludes the contribution of post peak energy, the Z-pinning mode II efficiency in terms of absorbed energy dropped slightly with loading rate, but this rate dependence is notably less significant than that in the mode I case. The mode II energy enhancement of Z-pins is also more modest in comparison with the mode I performance, with the quasi-static value of $4.01 \mathrm{~kJ} / \mathrm{m}^{2}(1.44$ $\mathrm{kJ} / \mathrm{m}^{2}$ without post-peak energy) and the dynamic value of $1.57 \mathrm{~kJ} / \mathrm{m}^{2}$ for the $2 \%$ volume fraction, comparing to the original mode II delamination toughness of $\sim 0.8 \mathrm{~kJ} / \mathrm{m}^{2}$ [36]. 
The study here showed that high loading rate characterization is essential, as quasi-static experiments are not necessarily a conservative estimation for the dynamic performance in Z-pinned laminates.

\section{Conclusions}

The performance of Z-pins in resisting dynamic mode I and mode II delamination has been investigated at a range of loading rates. A split Hopkinson bar system was used for the application of high loading rate to Z-pinned laminates. For quasi-static tests a universal testing machine was used. The unstable failure process in quasi-static tests was captured with a high frequency data acquisition method.

The inertia effects in the dynamic tests and the unstable fracture of quasi-static mode II tests were evaluated by means of finite element analysis. It was found that the inertia force should be deducted from the force measured directly from test systems.

The Z-pin efficiency in resisting mode I delamination decreased with increasing loading rate, which was caused by several factors: the friction force that is dependent on fracture surface, pin misalignment and the inertia effect involved in Z-pin bending during dynamic tests. The non-linear increase in bridging force during quasi-static pin pull-out was attributed to the pin misalignment. This conclusion was supported by the analysis of the failure surface of the holes vacated by pins after failure.

The Z-pin failed in a brittle manner during mode II tests. The maximum bridging force was not significantly influenced by the loading rate. The energy dissipated in this failure process was much lower than that in mode I tests, and decreased with increasing loading rate. This influence of loading rate was not conclusive, due the strong influence of frictional forces after pin rupture in the quasi-static tests. 


\section{Acknowledgements}

This research was supported by EPSRC funding in the UK (EP/M012905/1). The research data are available at ... (Permanent DOI to be provided after review)

\section{References}

[1] Davies GAO, Zhang X. Impact damage prediction in carbon composite structures. Int J Impact Eng. 1995;16:22.

[2] Schoeppner GA, Abrate S. Delamination threshold loads for low velocity impact on composite laminates. Composites Part A: Applied Science and Manufacturing. 2000;31:13.

[3] Mouritz AP. Review of z-pinned composite laminates. Composites Part A: Applied Science and Manufacturing. 2007;38(12):2383-2397.

[4] Cartié DDR, Dell'Anno G, Poulin E, Partridge IK. 3D reinforcement of stiffenerto-skin T-joints by Z-pinning and tufting. Engineering Fracture Mechanics. 2006;73(16):2532-2540.

[5] Dai S-C, Yan W, Liu H-Y, Mai Y-W. Experimental study on z-pin bridging law by pullout test. Composites Science and Technology. 2004;64(16):2451-2457.

[6] Cartié DDR, Cox BN, Fleck NA. Mechanisms of crack bridging by composite and metallic rods. Composites Part A: Applied Science and Manufacturing. 2004;35(11):1325-1336.

[7] Rugg KL, Cox BN, Massabo R. Mixed mode delamination of polymer composite laminates reinforced through the thickness by Z-fibers. Composites Part A: Applied Science and Manufacturing. 2002;33. 
[8] Rugg K. L CBN, Sherrick G. O. Damage mechanisms for angled throughthickness rod reinforcement in carbon-epoxy laminates. Composites Part A: Applied Science and Manufacturing. 1998;29A.

[9] M'Membe B, Gannon S, Yasaee M, Hallett SR, Partridge IK. Mode II delamination resistance of composites reinforced with inclined Z-pins. Mater Design. 2016;94:565-572.

[10] Yasaee M, Lander JK, Allegri G, Hallett SR. Experimental characterisation of mixed mode traction-displacement relationships for a single carbon composite Z-pin. Composites Science and Technology. 2014;94:123-131.

[11] Cui H, Li Y, Koussios S, Zu L, Beukers A. Bridging micromechanisms of Z-pin in mixed mode delamination. Composite Structures. 2011;93(11):11.

[12] Pegorin F, Pingkarawat K, Daynes S, Mouritz AP. Influence of z-pin length on the delamination fracture toughness and fatigue resistance of pinned composites. Composites Part B: Engineering. 2015;78:298-307.

[13] Yasaee M, Bigg L, Mohamed G, Hallett SR. Influence of Z-pin embedded length on the interlaminar traction response of multi-directional composite laminates. Mater Design. 2017;115:26-36.

[14] Allegri G, Yasaee M, Partridge IK, Hallett SR. A novel model of delamination bridging via Z-pins in composite laminates. International Journal of Solids and Structures. 2014;51(19-20):3314-3332.

[15] Bianchi F, Zhang X. Predicting mode-II delamination suppression in z-pinned laminates. Composites Science and Technology. 2012;72(8):924-932. 
[16] Cox BN. Snubbing effects in the pullout of a fibrous rod from a laminate. Mech Adv Mater Struc. 2005;12(2):85-98.

[17] Gerlach R, Siviour CR, Petrinic N, Wiegand J. Experimental characterisation and constitutive modelling of RTM-6 resin under impact loading. Polymer. 2008;49(11):2728-2737.

[18] Koerber H, Xavier J, Camanho PP. High strain rate characterisation of unidirectional carbon-epoxy IM7-8552 in transverse compression and in-plane shear using digital image correlation. Mechanics of Materials. 2010;42(11):1004-1019.

[19] Quaresimin M, Ricotta M, Martello L, Mian S. Energy absorption in composite laminates under impact loading. Composites Part B: Engineering. 2013;44(1):133-140.

[20] Abrate S. Impact on Laminated Composite Materials. Applied Mechanics Reviews. 1991;44(4):46.

[21] Zhang X, Hounslow L, Grassi M. Improvement of low-velocity impact and compression-after-impact performance by z-fibre pinning. Composites Science and Technology. 2006;66(15):2785-2794.

[22] Isa MD, Feih S, Mouritz AP. Compression fatigue properties of z-pinned quasiisotropic carbon/epoxy laminate with barely visible impact damage. Composite Structures. 2011;93(9):2269-2276.

[23] Liu H, Yan W, Yu X, Mai Y. Experimental study on effect of loading rate on mode I delamination of z-pin reinforced laminates. Composites Science and Technology. 2007;67(7-8):1294-1301.

[24] Schlueter AM. An Experimental Study of Rate Effects on Mode I Delamination of Z-pinned Composite. Purdue University, PhD Thesis, 2012. 
[25] Schlueter A, Parab ND, Chen W. Loading rate effect on mode I delamination of Z-pinned composite laminates. the 2013 Annual Conference on Experimental and Applied Mechanics, vol. 1: Springer; 2013. p. 8.

[26] Sridhar N, Massabo R, Cox BN, Beyerlein IJ. Delamination dynamics in through-thickness reinforced laminates with application to DCB specimen. International Journal of Fracture. 2002;118:26.

[27] Cui H, Li Y, Koussios S, Beukers A. Mixed mode cohesive law for Z-pinned composite analyses. Comp Mater Sci. 2013;75:60-68.

[28] Gerlach R, Kettenbeil C, Petrinic N. A new split Hopkinson tensile bar design. Int J Impact Eng. 2012;50:63-67.

[29] Miao Y-G, Li Y-L, Liu H-Y, Deng Q, Shen L, Mai Y-W, et al. Determination of dynamic elastic modulus of polymeric materials using vertical split Hopkinson pressure bar. International Journal of Mechanical Sciences. 2016;108-109:188-196.

[30] Zhao H. Material behaviour characterisation using SHPB techniques, tests and simulations. Comput Struct. 2003;81(12):1301-1310.

[31] Cui H, Thomson D, Pellegrino A, Wiegand J, Petrinic N. Effect of strain rate and fibre rotation on the in-plane shear response of $\pm 45^{\circ}$ laminates in tension and compression tests. Composites Science and Technology. 2016;135:106-115.

[32] Knopp A, Scharr G. Effect of z-pin surface treatment on delamination and debonding properties of z-pinned composite laminates. Journal of Materials Science. 2013;49(4):1674-1683. 
[33] Zhang B, Allegri G, Yasaee M, Hallett SR. Micro-mechanical finite element analysis of Z-pins under mixed-mode loading. Composites Part A: Applied Science and Manufacturing. 2015;78:424-435.

[34] Meo M, Achard F, Grassi M. Finite element modelling of bridging micromechanics in through-thickness reinforced composite laminates. Composite Structures. 2005;71(3-4):383-387.

[35] Czabaj MW, Ratcliffe JG. Comparison of intralaminar and interlaminar mode I fracture toughnesses of a unidirectional IM7/8552 carbon/epoxy composite. Composites Science and Technology. 2013;89:15-23.

[36] O'Brien TK, Johnston WM, Toland GJ. Mode II Interlaminar Fracture Toughness and Fatigue Characterization of a Graphite Epoxy Composite Material. NASA/TM-2010-216838, Hampton, Virginia, US: NASA Langley Research Center; 2010. 


\section{Figure captions}

Fig.1 Z-pin distribution in each specimen

Fig. 2 Mode I and mode II test configuration

Fig.3. (a) quasi-static and (b) dynamic experimental configuration

Fig.4. Finite element model for evaluating the inertia effect

Fig.5 Correction of inertia effect in the numerical simulations of (a) dynamic test configuration and (b) quasi-static test configuration

Fig.6 Failure process of Z-pins in mode I tests

Fig.7 (a) Bridging force and the displacement in dynamic test; (b) bridging-response at different loading rate

Fig.8 Failure surface of Z-pins: (a) original Z-pin; (b) Z-pin after quasi-static test; (c) Z-pin after dynamic test $(5.5 \mathrm{~m} / \mathrm{s})$

Fig. 9. Failure surface of holes after pin pull-out: (a) illustration of pull-out of Z-pins; (b) failure surface from quasi-static test; (c) failure surface from dynamic test $(5.5 \mathrm{~m} / \mathrm{s})$

Fig.10. The failure modes in shear tests

Fig.11. (a) Bridging force and energy dissipation in quasi-static mode II test; (b) velocity of fixtures measured with DIC in dynamic test; (c) original and corrected bridging force in dynamic test; (d) comparison of Z-pin bridging response in both quasistatic and dynamic tests

Fig.12. Failure modes of Z-pins in shear tests; (a) $0.01 \mathrm{~mm} / \mathrm{s}$, (b) $1.5 \mathrm{~m} / \mathrm{s}$ 
Fig.13 Energy dissipation in (a) mode I and (b) mode II failure of Z-pins 
2017-04-13

\title{
Bridging mechanisms of
}

\section{through-thickness reinforcement in dynamic mode I\&II delamination}

\author{
Cui, Hao
}

\section{Elsevier}

Hao Cui, Mehdi Yasaee, Gordon Kalwak, Antonio Pellegrino, Ivana K. Partridge, Stephen R. Hallett, Giuliano Allegri, Nik Petrinic, Bridging mechanisms of through-thickness reinforcement in dynamic mode I\&II delamination, Composites Part A: Applied Science and Manufacturing, Volume 99, August 2017, pp198-207

http://dx.doi.org/10.1016/j.compositesa.2017.04.009

Downloaded from Cranfield Library Services E-Repository 